\title{
8 AS REPRESENTAÇÕES SOCIAIS DO SUICÍDIO EM ADOLESCENTES: SCOPING REVIEW'1
}

\author{
| Rosa Simões²; José Carlos Santos³; Maria Júlia Martinhơ $\mid$
}

\section{RESUMO}

INTRODUÇÃO: O suicídio é um fenómeno complexo, multifatorial e uma das principais causas de morte potencialmente evitáveis. Melhorar a compreensão deste fenómeno, pelo conhecimento das representações sociais do suicídio em adolescentes, contribuirá para a intervenção psicoterapêutica em enfermagem.

OBJETIVO: Mapear as representações sociais do suicídio em adolescentes.

MÉTODOS: Scoping review com base nos princípios preconizados pelo Joanna Briggs Institute. Efetuada pesquisa na plataforma Biblioteca do conhecimento online, no Repositório Científico de Acesso Aberto de Portugal e no Banco de teses da Coordenação de Aperfeiçoamento de Pessoal de Nível Superior. Selecionados artigos em texto integral, publicados e não publicados, nos últimos 10 anos à data da pesquisa, em português, inglês e espanhol. Dois revisores independentes realizaram a análise de relevância dos artigos, extração e síntese dos dados.

RESULTADOS: Selecionados quatro estudos em que as representações sociais do suicídio dos adolescentes apontam para o suicídio como um ato egoísta, cobarde, impulsivo, irrefletido e de fuga, mas também como um ato de desespero e fraqueza, e uma estratégia de resolução dos problemas.

CONCLUSÕES: As representações sociais do suicídio em adolescentes diferem entre estudos, verificando-se escassez de estudos internacionais e a utilização de diferentes metodologias e diferentes instrumentos de avaliação não sendo por isso possível a comparação de resultados. Reforça-se a necessidade de promover investigação nesta área e contribuir para a adaptação de estratégias de prevenção do suicídio.

\section{PALAVRAS-CHAVE: Scoping review; Suicídio; Adolescente; Prevenção primária}

\section{RESUMEN}

"Las representaciones sociales del suicidio en adolescentes: Scoping review"

INTRODUCCIÓN: El suicidio es un fenómeno complejo y multifactorial y una de las principales causas de muerte potencialmente prevenibles. Mejorar la comprensión de este fenómeno, por el conocimiento de las representaciones sociales del suicidio en adolescentes, contribuirá a la intervención psicoterapéutica en enfermería.

OBJETIVO: Mapear las representaciones sociales del suicidio en adolescentes.

METODOLOGÍA: Scoping review basado en los principios preconizados por el Joanna Briggs Institute. Investigación llevada a cabo en la plataforma Biblioteca de conocimiento online, los científicos de libre acceso al repositorio de Portugal y Banco tesis de Coordinación de Mejora Personal de Nivel Superior. Artículos seleccionados en texto completo, publicados y no publicados, en los últimos 10 años en el momento de la encuesta, en portugués, inglés y español. Dos revisores independientes realizaron el análisis de relevancia de los artículos, la extracción y síntesis de los datos. RESULTADOS: Seleccionados cuatro estudios en los que las representaciones sociales del suicidio adolescente apuntan al suicidio como un acto egoísta, cobarde, impulsivo, irreflexivo y fugaz, pero también como un acto de desesperación y debilidad y una estrategia de resolución de problemas. CONCLUSIONES: Las representaciones sociales del suicidio en adolescentes difieren entre estudios, verificándose escasez de estudios internacionales y la utilización de diferentes metodologías e diferentes instrumentos de evaluación no por lo que es posible la comparación de resultados. Se refuerza la necesidad de promover investigación en este ámbito y contribuir a la adaptación de estrategias de prevención del suicidio.

\section{DESCRIPTORES: Scoping review; Suicidio; Adolescente; Pre- vención primaria}

\section{ABSTRACT \\ "The social representations of suicide in adolescents: Scoping review"}

BACKGROUND: Suicide is a complex and multifactorial phenomenon and one of the main potentially preventable causes of death. Improving the understanding of this phenomenon, through the knowledge of the social representations of suicide in adolescents, will contribute to the psychotherapeutic intervention in nursing.

AIM: To map the social representations of suicide in adolescents.

METHODS: Scoping review based on principles advocated by Joanna Briggs Institute. A research was carried out on the Biblioteca do conhecimento online platform, in the Open Access Scientific Repository of Portugal and in the Thesis Bank of the Coordination for the Improvement of Higher Education Personnel. Selected full-text articles, published and unpublished, in the last 10 years at the time of the research, in Portuguese, English and Spanish. Two independent reviewers performed the analysis of article relevance, extraction and synthesis of the data.

RESULTS: Four studies were selected in which the social representations of adolescent suicide point to suicide as a selfish, cowardly, impulsive, thoughtless and fleeting act, but also as an act of despair and weakness and a problem-solving strategy.

CONCLUSIONS: The social representations of suicide in adolescents differ between studies, with a shortage of international studies, and the use of different methodologies and different evaluation tools, so that results can not be compared. It reinforces the need to promote research in this area and to contribute to the adaptation of suicide prevention strategies.

\section{KEYWORDS: Scoping review; Suicide; Adolescent; Primary prevention}

Submetido em 30-09-2019

Aceite em 23-12-2019

1 Artigo extraído de Tese de Doutoramento em Ciências de Enfermagem, do Instituto de Ciências Biomédicas Abel Salazar, Universidade do Porto.

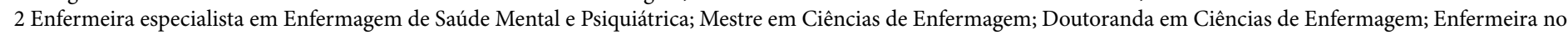
Centro Hospitalar e Universitário de Coimbra, EPE, Serviço de Urgência, Coimbra, Portugal, 400@chuc.min-saude.pt

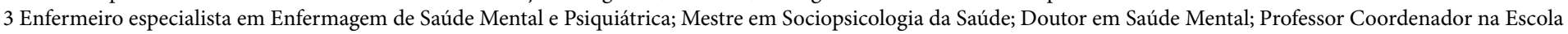
Superior de Enfermagem de Coimbra, Coimbra, Portugal,jcsantos@esenfc.pt

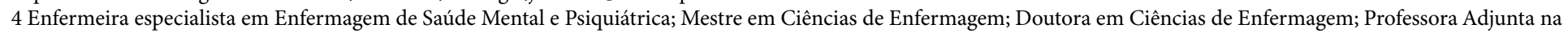
Escola Superior de Enfermagem do Porto, Porto, Portugal, julia@esenf.pt

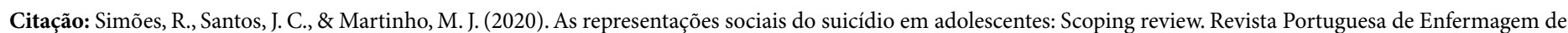
Saúde Mental (23), 54-61. 


\section{INTRODUÇÃO}

O suicídio é a segunda causa de morte nos adolescentes dos 15 aos 19 anos de idade sendo mesmo responsável por $85 \%$ das mortes nesta faixa etária, em todo o mundo (World Health Organization [WHO], 2014). E atualmente existem evidências relativas a um aumento do número de casos (World Health Organization, 2014).

Estima-se ainda, que por cada suicídio consumado em adolescentes, ocorram entre 100 e 200 tentativas de suicídio (Bertolote \& Fleischmann, 2009), o que nos alerta para a natureza repetitiva destes comportamentos, tornando-os nos maiores preditores de comportamentos futuros e de eventual morte por suicídio $(\mathrm{Ou}-$ grin \& Latif, 2011). Em congruência com esta premissa, as evidências disponíveis apontam para que nos nove anos após uma primeira tentativa de suicídio, 3 a $12 \%$ dos indivíduos terão morrido por suicídio (Owens, Horrocks, \& House, 2002).

O suicídio consumado é definido como um ato suicida, levado a cabo pelo indivíduo com intenção de pôr termo à vida (Direção-Geral de Saúde [DGS], 2013) e nestes atos suicidas incluem-se também a tentativa de suicídio, enquanto ato levado a cabo por um indivíduo e que visa a sua morte, mas que, por razões diversas, resulta frustrado (DGS, 2013). Já a ideação suicida é definida como os pensamentos e cognições sobre o acabar com a própria vida e os comportamentos autolesivos reportam-se aos comportamentos sem intencionalidade suicida, mas envolvendo atos autolesivos intencionais (DGS, 2013).

Ainda em termos conceituais, os comportamentos suicidários em adolescentes englobam os atos suicidas, já que sendo estes, mais que um comportamento autolesivo, podem estar presentes desde o suicídio consumado até à ideação suicida, passando pelos graus intercalares da tentativa de suicídio e dos comportamentos autolesivos (Saraiva, 2010). Estas especificidades, nesta faixa etária, levantam inúmeras questões em termos de prevenção, tornando-se por isso fundamental aumentar a compreensão da perceção que os adolescentes têm do suicídio, estudando as representações sociais deste fenómeno.

Para tal, é fundamental considerar que as representações sociais, enquanto sistemas de interpretação dependem da pertença categorial do sujeito, dos seus contextos de vida e de interação, do seu lugar na estrutura social e evidenciam-se como uma forma de ler o real, dando sentido à vida dos grupos e organizando-os em torno de interesses comuns (Cabecinhas, 2009).
Permitem ainda regular a relação com os outros e orientam o comportamento, intervindo em processos tão variados como a construção de identidade pessoal, o comportamento intra e intergrupal, as ações de resistência e de mudança social (Cabecinhas, 2009; Sampaio et al., 2000). Desta forma, os comportamentos adotados pelo indivíduo são resultantes do modo como este os representa socialmente, atribuindo-lhes um significado pessoal com repercussões na sua vida. Assim, a investigação em representações sociais permite entender a forma como os indivíduos apreendem o mundo envolvente, num esforço para o compreender e assim ajudar a resolver os seus problemas, sejam eles de ordem emocional, existencial, relacional, ou de qualquer outra ordem.

Com maior conhecimento e compreensão das representações sociais do suicídio em adolescentes, poderemos contribuir para o desenvolvimento de programas de prevenção e intervenção psicoterapêutica mais congruentes, a implementar pelo enfermeiro especialista em enfermagem de saúde mental e psiquiátrica. Este tipo de programa insere-se no âmbito das competências específicas do enfermeiro especialista em enfermagem de saúde mental e psiquiátrica já que este presta cuidados psicoterapêuticos, sócio terapêuticos, psicossociais e psicoeducacionais, à pessoa ao longo do ciclo de vida, mobilizando o contexto e dinâmica individual, familiar de grupo ou comunitário, de forma a manter, melhorar e recuperar a saúde (Ordem dos Enfermeiros, 2018).

\section{MÉTODOS}

Foi efetuada uma scoping review que seguiu a metodologia preconizada pelo Joanna Briggs Institute (2017) por ser uma abordagem projetada para sintetizar, de forma mais efetiva e rigorosa, a evidência científica e por ser o tipo de revisão que assume como principais objetivos: mapear as evidências existentes subjacentes a uma área de pesquisa, identificar lacunas na evidência existente, constituir um exercício preliminar que justifique e informe a realização de uma revisão sistemática da literatura (Peters et al., 2017).

Para estes autores, uma das particularidades desta metodologia é que a mesma não visa analisar a qualidade metodológica dos estudos incluídos, dado que o seu objetivo não é encontrar a melhor evidência científica, mas sim, mapear a evidência científica existente. Como a scoping review é exploratória, todos os resultados encontrados acerca do tema devem ser incluídos utilizando a estratégia participants, concept e context (PCC). 
Nesse sentido, a presente revisão tem como critérios de inclusão: adolescentes com idade entre os 10 e os 19 anos de idade; centrados no tema representações sociais do suicídio; em contexto clínico ou não clínico; em relação ao desenho dos estudos incluídos foram os estudos primários, tanto qualitativos e quantitativos.

Foram definidas as seguintes questões de pesquisa: "quais as representações sociais dos adolescentes acerca do suicídio?" e "quais as metodologias e instrumentos utilizados para conhecer as representações sociais dos adolescentes acerca do suicídio?”.

\section{Estratégia de pesquisa}

A pesquisa decorreu de 28 a 31 de janeiro de 2019 e incluiu estudos publicados e não publicados. Foi efetuada na plataforma Biblioteca do conhecimento online (Bon), com acesso a múltiplas bases de dados: Medline, SciELO, CINAHL Complete, Cochrane Collection Plus, Nursing \& Allied Health Collection: Expanded e Medic Latina; e, para a pesquisa de estudos não publicados, recorremos ao Repositório Científico de Acesso Aberto de Portugal (RCAAP) e ao Banco de teses da Coordenação de Aperfeiçoamento de Pessoal de Nível Superior (CAPES).

Os descritores de pesquisa utilizados foram: "social representation", “interpretation", "meaning”, "suicide”, “attempted suicide", "suicidal ideation", "adolescent", "teenager", "young" e "adolescence".

$\mathrm{Na} \mathrm{B}$-on realizaram-se as seguintes conjugações boleanas: (SU Social representation) OR (SU interpretation) OR (SU meaning) AND (SU suicide) OR (SU attempted suicide) OR (SU suicidal ideation) AND (SU adolescent) OR (SU teenager) OR (SU young) OR (SU adolescence). No RCAAP e no Banco de teses da CAPES foram pesquisados os termos "social representation" $\mathrm{e}$ "suicide", em título. Como limitadores foi considerado o período de publicação compreendido entre um de janeiro de 2009 e 31 de dezembro de 2018 (últimos 10 anos, à data da pesquisa), em português, inglês e espanhol e artigos em texto integral.

Foram analisadas todas as referências bibliográficas de todos os artigos e relatórios identificados, a fim de identificar estudos adicionais de interesse para este estudo, tendo sido recuperados alguns resumos e artigos em texto integral, mas não se verificou a inclusão de qualquer outro artigo, por não cumprirem os critérios de inclusão definidos. A relevância dos artigos para a revisão foi analisada por dois revisores independentes, com base nas informações fornecidas no título e resumo.
Sempre que os revisores tiveram dúvidas sobre a relevância de um estudo a partir do resumo, o artigo completo foi recuperado, assim como para todos os restantes estudos que cumpriam os critérios de inclusão.

Extração de dados

Os dados foram extraídos por dois revisores independentes, usando um instrumento desenvolvido pelos investigadores, alinhado com o objetivo definido e a questão de revisão.

\section{RESULTADOS}

Através desta estratégia de pesquisa foram encontradas 608 referências, sendo 574 localizadas na plataforma B-on, 20 no RCAAP e 14 no Banco de teses da CAPES, potencialmente relevantes (Figura 1). Após exclusão dos duplicados e dos registos que não apresentavam texto integral restaram 525 registos. Destes, 59 foram alvo de leitura dos títulos, selecionando-se 24 registos para leitura dos resumos. Por fim, selecionamos 14 registos para avaliação da elegibilidade, tendo sido incluído nesta revisão quatro destes estudos por cumprirem os critérios de inclusão (Oliveira, 2009; Araújo, Vieira e Coutinho, 2010; Gonçalves, 2013; Cabral, 2015).

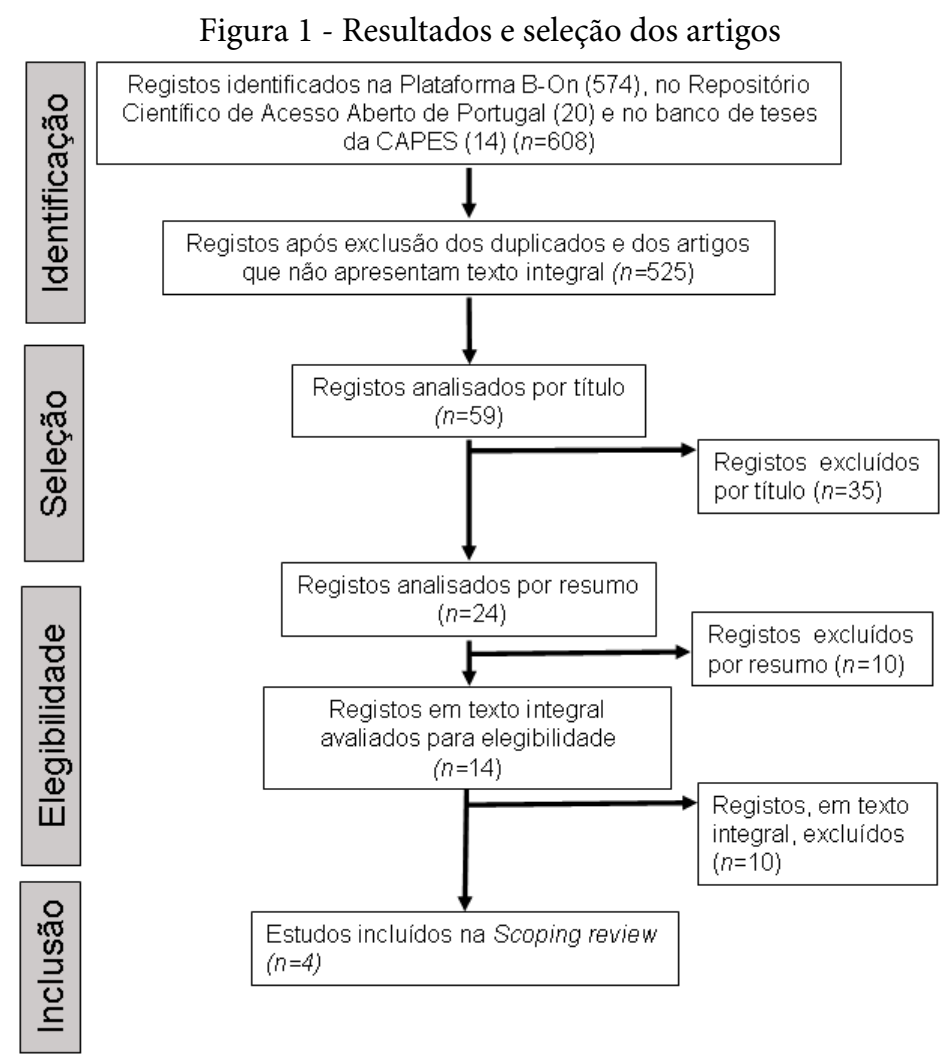

Tal como previsto numa scoping review os resultados são apresentados em tabela (Tabela 1), e em narrativa. De modo a enquadrar os resultados obtidos com as questões norteadoras desta revisão. 
Os estudos incluídos encontram-se distribuídos entre 2009 e 2015 (Araújo et al., 2010; Cabral, 2015; Gonçalves, 2013; Oliveira, 2009). No que concerne à metodologia utilizada nos estudos incluídos, dois apresentam desenho quantitativo (Oliveira, 2009; Cabral, 2015), um desenho qualitativo (Gonçalves, 2013) e o outro estudo um desenho misto (Araújo et al., 2010).

No que se refere aos instrumentos utilizados, Cabral (2015) recorreu a um instrumento aferido para adolescentes, o Questionário Suicídio Juvenil - Representações Sociais dos Adolescentes de Henriques, Rothes, Vila, e Oliveira (2008). Oliveira (2009) construiu um instrumento específico, mas com base no questionário anteriormente mencionado e Araújo et al. (2010) construiu um questionário com recurso ao teste de associação de palavras. Em comum, nestes três estudos foi solicitado aos participantes que, de forma espontânea, escrevessem as palavras ou expressões, relativas a pensamentos, sentimentos, objetos ou acontecimentos, suscitados por frases-estímulo ou indutores relativos ao suicídio. Nestes estudos foi utilizada a mesma técnica de análise dos dados: técnica de associação livre de palavras (Cabral, 2015; Oliveira, 2009; Araújo et al., 2010). Por outro lado, o estudo desenvolvido por Gonçalves (2013) utilizou um guião de entrevista.

Os participantes dos estudos foram adolescentes com idades entre os 12 e os 19 anos de idade, seguindo os limites definidos pela WHO (2019). Em três estudos, a amostra em estudo, encontrava-se em contexto não clínico, mais especificamente em contexto escolar (Oliveira, 2009; Araújo et al., 2010; Cabral, 2015), sendo que apenas um estudo foi realizado com uma população clínica em contexto de serviço de urgência, após admissão por tentativa de suicídio, tal como identificado na Tabela 1.

Todos os estudos incluídos encontram-se publicados em língua portuguesa, dois realizados em Portugal (Oliveira, 2009; Cabral, 2015) e dois no Brasil (Araújo et al., 2010; Gonçalves, 2013).

Seguindo a ordem de apresentação na Tabela 1 , constata-se que no estudo realizado por Cabral (2015), os resultados relativos aos significados atribuídos pelas adolescentes do género feminino centram-se no escape dos problemas (expressão mais referida), seguida de acabar com a própria vida, egoísmo e ato de desespero.

Quanto aos significados associados ao conceito de suicídio atribuídos pelos adolescentes do género masculino, destacam-se, como expressões mais mencionadas, o escape aos problemas, acabar com a própria vida, egoísmo e cobardia (Cabral, 2015).
Os adolescentes atribuem ao suicídio as causas: escape de problemas, bullying, problemas amorosos e fatores biológicos (Cabral, 2015).

Relativamente ao estudo desenvolvido por Araújo et al. (2010), a representação do comportamento suicidário traduz-se num ato de desespero e de fraqueza diante dos problemas descrevendo-o de forma ambígua, pois ao mesmo tempo em que é visto como algo mau, também é associado a uma sensação de alívio. Estas representações apoiam-se nos fatores desencadeantes do ato, focalizados na ausência de amor, no estado de sofrimento psíquico e desesperança do ser suicida (Araújo, et al., 2010). Os autores salientam que ao atribuir uma condição de fraqueza ao indivíduo, os participantes podem estar sinalizando dois sentidos diferentes e possíveis para o ser fraco: o primeiro como sinónimo de falta de força, de vigor, de solidez, de energia, provavelmente associado a um quadro depressivo, sendo uma manifestação predominantemente orgânica; e o segundo, significando foi atribuído à covardia, falta de obstinação, ou seja, o lado fraco do caráter de um ser, o que revela uma propensão a ceder a sugestões, imposições ou impressões e, nesse sentido, devendo ser encarada como manifestação psicológica (Araújo et al., 2010). Observa-se assim a existência de uma ambiguidade nas representações sociais do fenómeno suicídio, ao qual os adolescentes atribuíram valores positivos e negativos (Araújo et al., 2010). No estudo realizado por Oliveira (2009) entre os vários resultados salienta-se que, no que concerne aos significados associados ao conceito de suicídio, os adolescentes destacam, como expressões mais mencionadas: matar-se a si próprio, vida infeliz e ato irrefletido. Como causas de suicídio os adolescentes indicam os problemas familiares, vida infeliz e problemas escolares (Oliveira, 2009). Por seu lado, o estudo desenvolvido por Gonçalves (2013) numa amostra clínica, incluiu adolescentes admitidos num serviço de urgência hospitalar por tentativa de suicídio. O significado atribuído ao suicídio por estes adolescentes foi evidenciado como um ato impulsivo, sendo influenciado por inúmeros sentimentos negativos, associados a determinantes como drogas e desestruturação familiar. Para estes adolescentes os comportamentos suicidários foram precedidos de grandes sofrimentos associados a problemas mal resolvidos, representando a solução encontrada para fugir daquele sentimento de desesperança que os permeavam, e o recurso usado para amenizar a dor e aflição, tendo a impulsividade configurado o principal sentimento que antecedeu ao comportamento suicidário (Gonçalves, 2013). 
A ambiguidade na caraterização do comportamento surge aquando da perceção das implicações negativas do ato, pela constatação da ausência de mudanças nas suas vidas, e pela constatação do surgimento de atitudes hostis e exclusão do meio social por parte da sociedade em geral (Gonçalves, 2013).

Tabela 1 - Tipo de estudos, caraterísticas dos participantes e principais resultados

\begin{tabular}{|c|c|c|c|}
\hline Estudo & $\begin{array}{l}\text { Tipo de Estudo*/ Instru- } \\
\text { mento }^{\star *}\end{array}$ & $\begin{array}{l}\text { Caraterísticas dos partici- } \\
\text { pantes }\end{array}$ & $\begin{array}{l}\text { Resultados relativos às representações sociais dos } \\
\text { adolescentes }\end{array}$ \\
\hline Cabral, 2015 & $\begin{array}{l}{ }^{*} \text { Exploratório do tipo quanti- } \\
\text { tativo. } \\
{ }^{\star *} \text { Questionário Suicídio Juve- } \\
\text { nil - Representações Sociais } \\
\text { dos Adolescentes (Henriques, } \\
\text { Rothes, Vila, e Oliveira, 2008) }\end{array}$ & $\begin{array}{l}82 \text { Adolescentes do ensino } \\
\text { secundário, com idades com- } \\
\text { preendidas entre } 14 \text { e } 19 \text { anos } \\
\text { de idade. } \\
\text { População Não Clínica }\end{array}$ & $\begin{array}{l}\text { Por género, nas adolescentes o escape dos prob- } \\
\text { lemas é a expressão mais referida, seguida de } \\
\text { acabar com a própria vida, egoísmo e ato de deses- } \\
\text { pero. Já nos adolescentes destacam-se as expressões } \\
\text { escape dos problemas, acabar com a própria vida, } \\
\text { egoísmo e cobardia. } \\
\text { Como causas apontam o escape de problemas, } \\
\text { o bullying, os problemas amorosos e os fatores } \\
\text { biológicos. }\end{array}$ \\
\hline Araújo et al., 2010 & $\begin{array}{l}\text { *Abordagem multimétodo de } \\
\text { cunho qualitativo e quantita- } \\
\text { tivo. } \\
{ }^{*} \text { Questionário com Teste de } \\
\text { Associação de Palavras. }\end{array}$ & $\begin{array}{l}90 \text { Adolescentes do ensino } \\
\text { médio, com idade entre os } 14 \\
\text { e } 18 \text { anos. } \\
\text { População Não Clínica. }\end{array}$ & $\begin{array}{l}\text { Os comportamentos suicidários são representados } \\
\text { como um ato de desespero e fraqueza, mas também } \\
\text { ambiguamente como algo mau ou que traz alívio, } \\
\text { ancorados nos fatores desencadeantes do ato e } \\
\text { focalizados na ausência de amor, no estado de so- } \\
\text { frimento psíquico e na desesperança do ser suicida. }\end{array}$ \\
\hline Oliveira, 2009 & $\begin{array}{l}{ }^{*} \text { Exploratório do tipo quanti- } \\
\text { tativo. } \\
{ }^{\star *} \text { Questionário elaborado com } \\
\text { base no Questionário } \\
\text { Suicídio Juvenil - Represen- } \\
\text { tações Sociais de Profission- } \\
\text { ais, de Rothes e Henriques } \\
\text { (Rothes, 2006). }\end{array}$ & $\begin{array}{l}81 \text { Adolescentes com idades } \\
\text { entre os } 12 \text { e } 15 \text { anos }(\mathrm{M}= \\
13.06, \mathrm{DP}=0.11) \text { a frequentar- } \\
\text { em o } 7^{\circ}, 8^{\circ} \text { e } 9^{\circ} \text { ano em escola } \\
\text { do Norte de Portugal. } \\
\text { População Não Clínica. }\end{array}$ & $\begin{array}{l}\text { Para o significado associado ao conceito de } \\
\text { suicídio, destacam-se as expressões matar-se a si } \\
\text { próprio, vida infeliz e ato irrefletido. } \\
\text { Como causas para o suicídio são indicados os } \\
\text { problemas familiares, vida infeliz e problemas } \\
\text { escolares. }\end{array}$ \\
\hline Gonçalves, 2013 & $\begin{array}{l}{ }^{\star} \text { Exploratório, descritivo do } \\
\text { tipo qualitativo. } \\
{ }^{\star *} \text { Entrevistas semiestrutura- } \\
\text { das. }\end{array}$ & $\begin{array}{l}\text { Dois adolescentes com idades } \\
\text { entre os } 14 \text { e } 19 \text { anos de } \\
\text { idade, admitidos num serviço } \\
\text { de urgência por tentativa de } \\
\text { suicídio e suas famílias. } \\
\text { População Clínica. }\end{array}$ & $\begin{array}{l}\text { O suicídio é representado como ato impulsivo, } \\
\text { sendo influenciado por inúmeros sentimentos } \\
\text { negativos como a raiva e o desespero, associados } \\
\text { a determinantes como drogas e desestruturação } \\
\text { familiar. }\end{array}$ \\
\hline
\end{tabular}

\section{DISCUSSÃO}

O objetivo desta revisão foi analisar e mapear estudos que avaliassem as representações sociais do suicídio em adolescentes. As explicações que encontramos para este achado prendem-se com a sensibilidade do tema em estudo, em populações de adolescentes, já que foram identificados inúmeros estudos que foram excluídos essencialmente por os participantes não cumprirem o critério de inclusão referente à idade (entre os 10 e os 19 anos de idade), existindo vários artigos que se referem a adolescentes, mas que apresentam como limite de idade os 35 anos (Nikolay et al., 2014). A metodologia usada é mencionada de forma explícita no desenho dos estudos de Araújo et al. (2010) e Gonçalves (2013), sendo que nos restantes dois estudos, realizados por Cabral (2015) e Oliveira (2009), o desenho foi identificado pelos autores desta scoping review. Constata-se que não existe consenso acerca das metodologias a privilegiar. Neste sentido, alguns autores defendem que a metodologia qualitativa é a mais adequada para fomentar a compreensão do fenómeno suicídio e clarificar o que liga as atitudes dos participantes em relação à saúde, caraterísticas individuais e ambiente, facilitadores e barreiras no cuidado (Lester, 2010). Por outro lado, existem autores que contrapõem esta ideia por considerarem que a investigação em representações sociais faz apelo a metodologias variadas e não existe nenhuma metodologia que, por si só, seja suficiente para investigar estes complexos fenómenos (Cabecinhas, 2009). Ou seja, a investigação em representações sociais pode englobar uma pesquisa muito heterogénea e não prescritiva em termos de metodologia, sendo fundamental para o investigador centrar-se no processo de investigação e não em qualquer método específico (Oliveira e Amaral, 2007; Cabecinhas, 2009). 
Torna-se fundamental optar por técnicas que facilitem a apreensão das dimensões representacionais (nomeadamente, por métodos de recolha de dados que facilitem as respostas dos sujeitos) e auxiliem na procura e análise do que distingue ou aproxima essas representações, entre si, e em relação aos grupos sociais que constituem uma dada população (Cabecinhas, 2009), o que se verifica nos estudos selecionados e em que maioritariamente utilizaram a técnica de associação livre de palavras.

A justificação para a definição do limite temporal, mesmo não se localizando grande número de estudos, reside no facto de as representações sociais, serem influenciadas pelos contextos sociais, culturais e educacionais assim como pelos desafios e profundas mudanças ocorridas nestes âmbitos na última década, com esperado reflexo a nível individual, grupal, familiar e da sociedade em geral.

Evidencia-se a necessidade de investigação em populações clínicas que possibilitem comparação de resultados, assim como um melhor ajuste e adequação dos descritores usados na pesquisa, como forma de localização de estudos em língua inglesa. Este último aspeto mereceu grande reflexão e investimento, sem no entanto se conseguirem modificar os resultados, provavelmente pelas dificuldades na adaptação cultural dos termos da pesquisa.

Os adolescentes incluídos nos estudos analisados descrevem o suicídio como um ato irrefletido desencadeado por problemas familiares, vida infeliz e problemas escolares (Oliveira, 2009), mas também como um ato de desespero e fraqueza face aos problemas (ausência de amor, sofrimento psíquico, desesperança) em que podendo ser uma solução negativa pode, por outro lado, trazer alívio (Araújo et al., 2010). Corroborando com Oliveira (2009), os adolescentes incluídos no estudo desenvolvido por Gonçalves (2013) consideram o suicídio como um ato impulsivo e uma estratégia de fuga aos problemas, influenciado por sentimentos negativos associados ao consumo de drogas e à desestruturação familiar. Por outro lado, os adolescentes que participaram no estudo desenvolvido por Cabral (2015) representam o suicídio como um ato egoísta, cobarde e de desespero de acabar com a própria vida como estratégia de fuga aos problemas, dos quais destacam o bullying, os problemas amorosos e fatores biológicos.

Não obstante todos os estudos se referirem às representações sociais do suicídio em adolescentes, constatamos que os significados atribuídos ao suicídio denunciam algumas ambiguidades que podem ser explicadas pela complexidade do fenómeno associada às influências sociais, culturais e educacionais que afetam as representações sociais dos adolescentes, mas também pela heterogeneidade das populações estudadas. Relativamente aos adolescentes que integram a população clínica tornase evidente a importância do papel da família face às grandes transformações vividas pela adolescência sendo caracterizada como apoio e âncora. As histórias de vida também são marcantes e maioritariamente caraterizadas por separações e sofrimentos precoces, havendo ausência de diálogo, do acompanhamento e do estímulo dos pais, sendo a desestruturação familiar evidente nos discursos dos adolescentes e considerada um agente motivador para o comportamento suicidário. Por esse motivo, foi concluído neste estudo que a família exerce papel fundamental na prevenção do comportamento suicidário em adolescentes (Gonçalves, 2013).

Verifica-se que é unânime o facto de os adolescentes associarem uma crítica negativa ao suicídio, traduzida pelas expressões ato irrefletido, ato impulsivo, ato de fraqueza e desespero, estratégia de fuga, ato egoísta e cobarde (Oliveira, 2009; Araújo et al., 2010; Gonçalves, 2013; Cabral, 2015). Da mesma forma que é consensual a justificação do ato pela necessidade de fuga aos problemas intoleráveis, pelo desespero e sofrimento que causam. Os problemas familiares, escolares e amorosos, mas também a ausência de sentido da vida, o consumo de drogas e os fatores biológicos são apontados como as principais causas. Para Araújo et al. (2010) existem diferenças significativas nas representações sociais do suicídio por adolescentes, com ou sem ideação suicida, sendo que os adolescentes que não evidenciam ideação suicida são os que demonstram maior rejeição ao comportamento suicidário e atribuição de caráter negativo; por sua vez, os adolescentes com ideação suicida indicam maior familiaridade e aceitação do tema.

\section{CONCLUSÕES}

Realizamos uma scoping review com base nos princípios preconizados pelo Joanna Briggs Institute que nos permitiu atingir os objetivos delineados.

Constatamos a escassez de investigação desenvolvida sobre este tema e a escassez de estudos internacionais nos últimos anos, em particular com esta população. Assim como a utilização de diferentes metodologias e diferentes instrumentos de avaliação o que não permite a comparação alargada de resultados. 
As metodologias foram diversas, assim como os instrumentos de recolha de dados, mas verifica-se algum consenso na utilização da técnica de análise dos dados (técnica de associação livre de palavras) e que se justifica por ser uma técnica facilitadora da apreensão das dimensões representacionais por facilitar as respostas dos sujeitos, sendo um aspeto importante a reter e replicar em estudos futuros.

Quanto às limitações reportadas nos estudos incluídos e de modo a fornecer informações para estudos futuros, primários ou revisões sistemáticas, facilitando a comparação dos resultados, podemos acrescentar que estão maioritariamente relacionadas com: o tamanho da amostra, a técnica de amostragem, a homogeneidade da amostra, o contexto escolar, assim como o prévio conhecimento dos adolescentes de que o principal objetivo do estudo era a recolha de representações sociais sobre o suicídio. A ausência de referência a limitações nos estudos publicados e o recurso a instrumentos previamente validados também se nos afigura determinante no reforço das evidências.

Como limitações desta revisão destacamos o facto de se ter determinado como critérios de inclusão apenas artigos publicados em inglês, português e espanhol, já que artigos publicados noutros idiomas poderiam trazer dados relevantes para esta revisão. A selecção de estudos apenas em português, realizados em Portugal e no Brasil, também se nos afigura como uma limitação já que nos limita a análise de diferentes perspetivas socioculturais, no entanto este aspeto não foi passível de modificação apesar dos inúmeros esforços e alterações nos descritores e estratégias de pesquisa.

Estas limitações refletem-se no pequeno número de estudos, já que apesar do tema suicídio ser uma problemática muito estudada, tal não acontece no que se refere às representações sociais do suicídio em adolescentes dos 10 aos 19 anos e idade.

\section{IMPLICAÇÕES PARA A PRÁTICA CLÍNICA}

Quanto às implicações para a investigação, dada a importância amplamente descrita sobre a necessidade de conhecer as representações sociais do suicídio em adolescentes e, dessa forma, poder adaptar os programas de prevenção do suicídio.

Reforça-se a necessidade de promover mais investigação sobre este tema, com utilização de metodologias de cariz quantitativo e qualitativo, em populações não clínicas mas, essencialmente, em populações clínicas, de forma a aumentar os conhecimentos acerca da forma como estes adolescentes representam o fenómeno suicídio, melhorando a compreensão do significado atribuído e possibilitando a adaptação de intervenções de enfermagem em todos os níveis de prevenção. Futuros estudos deverão identificar de forma clara a metodologia selecionada, bem como as limitações dos mesmos e implicações para a prática clínica e investigação.

Por outro lado, dado que não é objetivo de uma scoping review avaliar a qualidade metodológica dos estudos incluídos, não são apresentadas recomendações para a prática.

A título conclusivo, entendemos como positivos os contributos deste trabalho já que consideramos que acrescentam dados à compreensão das representações sociais do suicídio em adolescentes, diferenciando-se as populações clínicas das populações não clínicas o que contribuirá para a adaptação das estratégias de prevenção e de intervenção psicoterapêutica em enfermagem em todos os níveis de prevenção do suicídio.

\section{REFERÊNCIAS BIBLIOGRÁFICAS}

Araújo, L. C., Vieira, K. F. L., \& Coutinho, M. P. L. (2010). Ideação suicida na adolescência: um enfoque psicossociológico no contexto do ensino médio. Psico-USF, 15(1), 47-57. Disponível em: http://www. scielo.br/scielo.php?script $=$ sci_arttext $\&$ pid $=$ S1413$82712010000100006 \& \operatorname{lng}=\mathrm{en} \& \mathrm{nrm}=\mathrm{iso}>$

Aromataris, E., \& Munn, Z. (2017). Joanna Briggs Institute Reviewer's Manual. The Joanna Briggs Institute. Disponível em: https://reviewersmanual.joannabriggs.org/

Bertolote, J., \& Fleischmann, A. (2009). A global perspective on the magnitude of suicide mortality. In D.Wasserman, \& C. Wasserman, The oxford textbook of suicidology and suicide prevention: a global perspective (pp. 91-98). Oxford: Oxford University Press.

Cabecinhas, R. (2009). Investigar representações sociais: Metodologias e níveis de análise. In M. M. Baptista (Eds.), Cultura: Metodologias e investigação (pp. 5166). Lisboa: Verso Edições.

Cabral, C. A. A. (2015). Representações Sociais do Suicídio na Adolescência (Dissertação/projeto de mestrado), Instituto Politécnico de Viseu, Portugal. Disponível em: http://repositorio.ipv.pt/bitstream/10400.19/2990 
Direção-Geral de Saúde. (2013). Plano nacional de prevenção do suicídio 2013/2017. Lisboa, Portugal: Direção-Geral de Saúde.

Gonçalves, N. D. (2013). Suicídio na adolescência: representações dos pacientes e suas famílias. (Dissertação de Mestrado), Universidade Estadual do Ceará, Brasil. Disponível em: https://sucupira.capes.gov.br/sucupira/ public/consultas/coleta/trabalhoConclusao/viewTrabalhoConclusao.jsf?popup $=$ true\&id_trabalho $=224253$

Henriques, M. R., Rothes, I., Vila, A., \& Oliveira, A.F. (2008). Questionário Suicídio juvenil - Representações sociais dos adolescentes. (Dissertação de Mestrado). Universidade do Porto, Portugal. Disponível em: https://repositorio-aberto.up.pt/bitstream/10216/105312/2/30244.pdf

Lester, D. (2010). Qualitative Research in Suicidology: Thoughts on Hjelmeland and Knizek's "Why we Need Qualitative Research in Suicidology". Suicidology Online, 1, 76-78. Disponível em: http://www.suicidologyonline.com/pdf/SOL-2010-1-76-78.pdf

Nikolay, D., Inna, B., Olesya, V., Elizaveta, B., Gennady, B., \& Inga, K. (2014). Self-murder and self-murderers in social representations of young Russians: An exploratory study. Psichologija, 50, 33-48. Disponível em: https:// psycnet.apa.org/record/2015-15903-003

Oliveira, A. F. S. S. (2009). Representações Sociais de Morte e de Suicídio em Adolescentes (Tese Mestrado). Universidade do Porto, Porto, Portugal. Retrieved from https://repositorio-aberto.up.pt/bitstream/10216/105312/2/30244.pdf

Oliveira, A., \& Amaral, V. (2007). A análise factorial de correspondências na investigação em psicologia: Uma aplicação ao estudo das representações sociais do suicídio adolescente. Análise Psicológica, 2(XXV), 271-293. Disponível em: http://www. scielo.mec.pt/scielo.php?script=sci_arttext\&pid $=$ S0870-82312007000200008

Ordem dos Enfermeiros. (2018). Regulamento de Competências Específicas do Enfermeiro Especialista em Enfermagem de Saúde Mental e Psiquiátrica: Regulamento n. ${ }^{\circ}$ 515/2018 - Diário da República n. ${ }^{\circ}$ 151/2018, Série II de 2018-08-07. Disponível em: https://www.ordemenfermeiros.pt/media/8739/2142721430.pdf
Ougrin, D., \& Latif, S. (2011). Specific psychological treatment versus treatment as usual in adolescents with self-harm: Systematic review and meta-analysis. Crisis, 32, 74-80. Disponível em: https://www.ncbi.nlm.nih. gov/pubmed/21616756

Owens, D., Horrocks, J., \& House, A. (2002). Fatal and non-fatal repetition of self-harm. A systematic review. British Journal of Psychiatry, 181, 193-199. Disponível em: http://bjp.rcpsych.org/content/bjprcpsych/181/3/193.full.pdf

Peters, M., Godfrey, C., McInerney, P., Baldini Soares, C., Khalil, H., Parker, D. (2017). Chapter 11: Scoping Reviews. In: Aromataris, E., \& Munn, Z. (Editors). Joanna Briggs Institute Reviewer's Manual. Disponível em: https://reviewersmanual.joannabriggs.org/

Rothes, I. (2006). Suicídio juvenil: Representações sociais dos médicos e dos psicólogos. (Dissertação de Mestrado). Universidade do Porto, Portugal. Disponível em: https://sigarra.up.pt/reitoria/pt/pub_geral.show_ file?pi_doc_id=21742

Sampaio, D., Oliveira, A., Vinagre, M., Pereira, M., Santos, N., \& Ordaz, O. (2000). Representações sociais do suicídio em estudantes do ensino secundário. Análise Psicológica, 2 (XVIII), 139-155. Disponível em: http://www.scielo.mec.pt/scielo.php?script=sci_ abstract\&pid $=$ S0870-82312000000200001\&lng=pt\&nr $\mathrm{m}=\mathrm{iso}$

Saraiva, C. B. (2010). Prefácio. In N. A. C. Moreira (Eds.), Suicídio nas Prisões (pp. 5-8). Porto: Legis Editora.

World Health Organization. (2014). Preventing suicide: a global imperative. Geneva: World Health Organization. Disponível em: http://www.who.int/ mental_health/suicide-prevention/exe_summary_english. pdf?ua $=1$

World Health Organization. (2019). Health for the world's adolescentes. Online report. Disponível em: https://www.who.int/maternal_child_adolescent/topics/adolescence/second-decade/en/ 


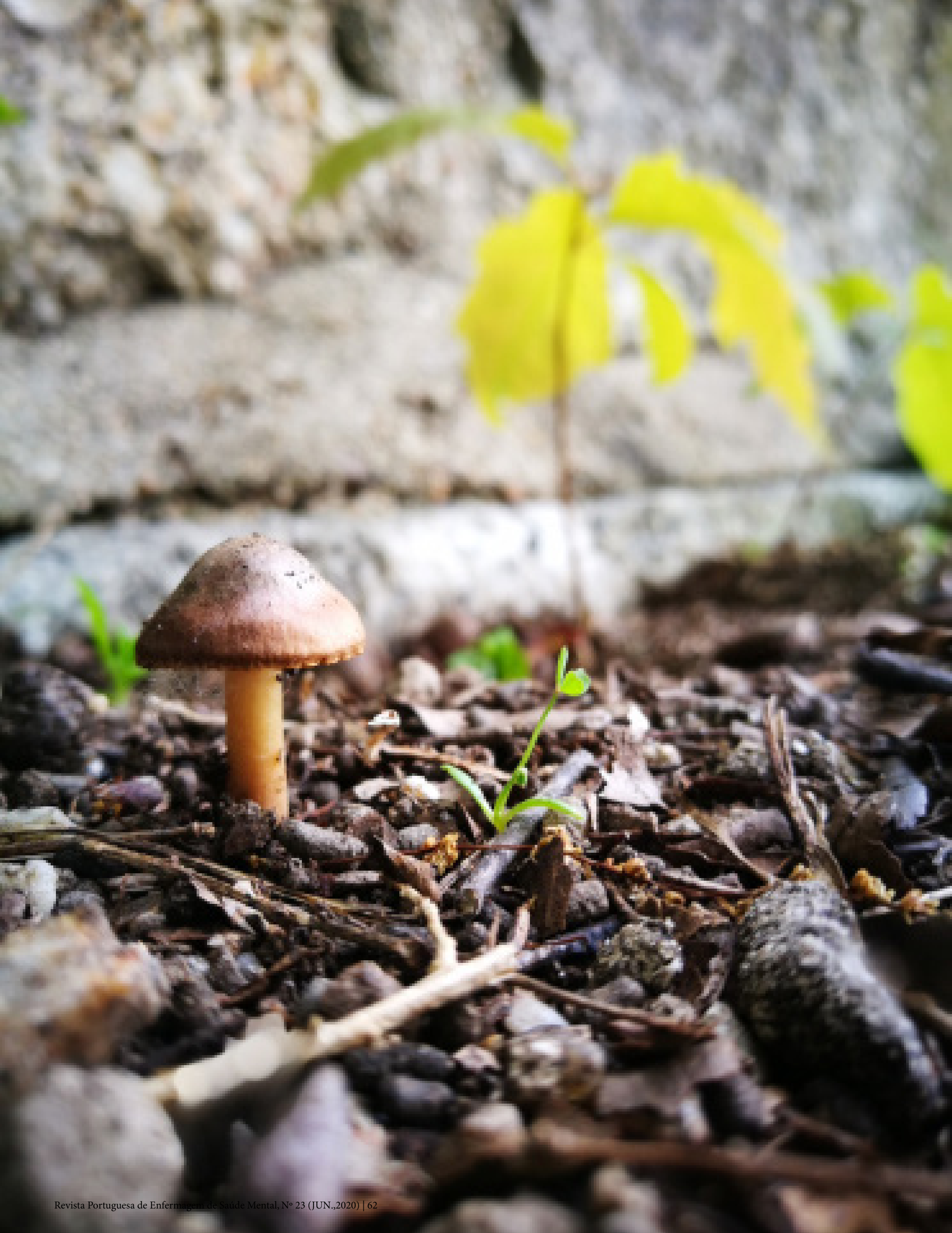

